Jpn. J. Med. Sci. Biol., 51, Suppl. 1, S101-S107, 1998

\title{
The Locus of Enterocyte Effacement Pathogenicity Island of Shiga Toxin-Producing Escherichia coli 0157:H7 and Other Attaching and Effacing $E$. coli
}

\author{
James B. Kaper
}

Center for Vaccine Development University of Maryland School of Medicine, Baltimore, MD, U.S.A.

\section{INTRODUCTION}

Shiga toxin-producing E. coli (STEC) O157:H7 and enteropathogenic $E$. coli (EPEC) produce a characteristic histopathology on intestinal epithelial cells known as attaching and effacing $(\mathrm{A} / \mathrm{E})$. The characteristics of this pattern includes effacement of epithelial cell microvilli and intimate adherence between the bacterium and the epithelial cell membrane. Marked cytoskeletal changes are seen directly beneath the adherent bacterium, including accumulation of polymerized actin, and the development of pedestal-like structures upon which the bacteria sit. Tzipori et al. (20) first reported the attaching and effacing histopathology in gnotobiotic piglets infected with STEC O157:H7 and it has since been reported in variety of animal species as well as with cultured epithelial cells infected with O157:H7 (reviewed in (14)). This pattern was first described for EPEC and it has been best characterized in this pathogen.

\section{Cellular response}

A major component of the cellular response to infection by STEC $\mathrm{O} 157: \mathrm{H} 7$ or other A/E pathogens is the accumulation of polymerized actin directly beneath the adherent bacteria. Increases in intracellular calcium levels have also been reported in response to infection by $\mathrm{A} / \mathrm{E}$ pathogens and this is accompanied by the release of inositol phosphates (reviewed in (8)). Activation of at least two serine/threonine kinases has been reported including protein kinase $\mathrm{C}$ and myosin light chain kinase (reviewed in (8)). Activation of tyrosine kinases has also been reported. With EPEC strain E2348/69, Rosenshine et al. (17) reported that binding of the bacterium to the epithelial cell resulted in phosphorylation on a 
tyrosine residue of a $90 \mathrm{kDa}$ protein in the epithelial cell. Recently, this protein has been shown to be of bacterial origin rather than eukaryotic origin and has been named Tir (see below). Interestingly, tyrosine phosphorylation of a $90 \mathrm{kDa}$ protein has not been found with $E$. coli O157:H7 but has been found with an STEC O26:H7 strain (2).

Beyond the individual enterocyte, there are additional host responses to infection by STEC 0157:H7 and EPEC. In monolayers of polarized epithelial cells such as Caco-2 or T84, infection with STEC O157:H7 or EPEC results in a decrease in the transepithelial resistance of the monolayers as a result of the opening of tight junctions $(16,25)$. With EPEC, adherence to epithelial cells activates the eukaryotic transcriptional factor NF-B, which in turn upregulates expression of IL-8 (19), leading to transmigration of polymorphonuclear leukocytes (PMNs) from the basolateral to apical sides of epithelial cells (18). In a rabbit model of infection with STEC O157:H7, PMN infiltration was blocked by the addition of antibodies to the leukocyte adhesion molecule CD18 (3) and inhibition of this inflammatory response led to a reduction in the diarrhea seen in this animal model.

\section{The LEE pathogenicity island}

Genes essential for production of the A/E phenotype were found by McDaniel et al. (12) to be located on a $35 \mathrm{~kb}$ region of the chromosome which they called the Locus of Enterocyte Effacement (LEE). The LEE was first described for EPEC strain E2348/69 but is also present in STEC 0157:H7 and other pathogens capable of producing the A/E histopathology (see below). The LEE is not found in normal flora $E$. coli, enterotoxigenic $E$. coli or other strains that cannot produce the A/E phenotype. The entire LEE of EPEC strain E2348/69 has been cloned on a single plasmid which when present in $E$. coli $\mathrm{K}-12$ is sufficient to produce the A/E phenotype (13). The $\mathrm{G}+\mathrm{C}$ content of the LEE $(38.3 \%)$ is much lower than that of the $E$. coli chromosome (50.8\%), suggesting that the E. coli LEE arose by horizontal gene transfer of this pathogenicity island from another species.

The LEE of EPEC E2348/69 (4) and STEC O157:H7 strain 933 (15) contains 41 open reading frames of greater than 50 amino acids. These genes are organized into three major regions with known functions. The middle region contains the eae gene which encodes intimin, a 94-97 $\mathrm{kDa}$ outer membrane protein that is an intestinal adherence factor. The importance of intimin as an adherence factor has been shown with isogenic eae mutants in volunteers experimentally infected with EPEC and in gnotobiotic piglets infected with STEC O157:H7 (reviewed in $(9,14)$ ). 
Also in this region is the tir gene which encodes a $78 \mathrm{kDa}$ protein that is translocated into the plasma membrane of eukaryotic cells infected with EPEC (10). Once inside the eukaryotic cell, the Tir protein is tyrosinephosphorylated and the apparent molecular weight increases to $90 \mathrm{kDa}$. In STEC O26:H-strains, the Tir protein has been called EspE and is similarly tyrosine phosphorylated upon translocation into epithelial cells (2). The translocated Tir protein serves as a receptor for the intimin protein on the surface of the bacterium (10).

Upstream of the eae and tir genes are genes encoding a type III protein secretion system $(5,6)$. Such type III systems have been described in Salmonella, Shigella, Yersinia, Pseudomonas, and Chlamydia spp as well as in several plant pathogens. For all of these pathogens, the type III secretion systems are responsible for the translocation of proteins from the bacterial cytoplasm to outside the bacterial cell where they can interact with the eukaryotic host cell. The proteins secreted by the type III systems are essential for pathogenesis of their respective species and are secreted without the use of a conventional signal peptide (leader sequence). For EPEC and STEC O157:H7, the genes encoding the type III secretion system are called esc; the esc genes encode proteins with homology to components of other type III systems. There are some additional genes involved in secretion of these proteins which show no homology to other type III systems and these genes are called sep (4).

Several proteins are secreted via the type III systems of EPEC and STEC including EspA (25 kDa), EspB (38 kDa), and EspD (40 kDa) and mutation of any one of the espABD genes abolishes the A/E phenotype (reviewed in (8)). The EspB protein is translocated into the eukaryotic cell where it is believed to be responsible for triggering the signal transduction events seen in the host cell in response to EPEC/STEC infection (24). The EspA protein has recently been shown by Knutton et al. (11) to form a filamentous appendage which connects the bacterial cell to the host cell and through which proteins such as EspB and Tir are translocated into the eukaryotic cell. The function of the EspD protein is currently unknown.

The complete DNA sequence of the LEE for EPEC strain E2348/69 (4) and STEC O157:H7 strain 933 (15) have been determined. The average nucleotide identity between these strains is $93.9 \%$ across the 41 shared genes (15). The esc genes encoding the type III secretion system are the most highly conserved and share 98 to $100 \%$ identity. Interestingly, those genes encoding proteins that interact with the host cells are less conserved with the eae genes sharing $87.23 \%$ identity, espB sharing $74 \%$ identity, espA sharing $84.63 \%$ identity and the tir genes 
sharing $66.48 \%$ identity.

\section{Distribution and chromosomal insertion of the LEE}

In addition to being present in EPEC and STEC O157:H7, the LEE is also present in a variety of $E$. coli strains associated with diarrhea in rabbits, calves, pigs, and dogs, strains of Hafnia alvei associated with diarrhea, and Citrobacter rodentium associated with colonic hyperplasia in mice (reviewed in (8)). The LEE is also present in many but not all STEC of serotypes other than O157:H7. (The presence of the LEE is usually determined by the detection of the eae gene.) The LEE is present in the majority of STEC strains associated with human disease but is not present in the majority of STEC strains isolated from animals or foods (reviewed in (7)). Johnson et al. (7) reviewed non-O157:H7 STEC strains and summarized the occurrence of the eae gene (and presumably the rest of the LEE) in outbreaks due to non-O157:H7 serotypes. STEC of serotypes $\mathrm{O} 145: \mathrm{H}-, \mathrm{O} 111: \mathrm{H}-$ (four outbreaks), O26:H11, O157:H-, O ?: $\mathrm{H} 19$, and O104:H21 have caused outbreaks of human disease and all but $\mathrm{O}$ ? :H19 and O104:H21 contained eae sequences.

In STEC O157:H7 and EPEC strain E2348/69, the LEE is inserted at minute 82 of the $E$. coli $\mathrm{K}-12$ chromosome, just downstream of the selC locus (12). This site is also the site of insertion of a pathogenicity island in uropathogenic $E$. coli. However, this insertion site is not the site of the LEE in all EPEC or STEC strains. Wieler et al. (23) found that the insertion of the LEE differed in relation to the clonal phylogeny of the strains, i.e., EPEC or STEC strains that evolved along distinct evolutionary lines as determined by sequence variation in housekeeping genes had a LEE insertion site that was consistent with these phylogenetic lineage of the strains. Thus, strains grouped into the EPEC1 or EHEC1 groups by Whittam (22) (determined on the basis of housekeeping gene sequence variation) had the LEE inserted in the selC locus. Strains that were grouped into EPEC2 or EHEC2 on the basis of housekeeping genes did not have the LEE inserted at minute 82 but at another site. Benkel et al. (1) recently showed that in many of these strains, the LEE was inserted at minute 94 on the $E$. coli $\mathrm{K}-12$ chromosome, at the pheU gene. Recent data from my laboratory indicates that the LEE may also be inserted at a third chromosomal site.

These results and other data suggests a likely evolutionary scenario for the emergence of STEC 0157:H7 (reviewed by Whittam(21)). This serotype emerged from an EPEC strain, perhaps of the O55:H7 serotype, to which $0157: \mathrm{H} 7$ is closely related on the basis of housekeeping gene variation. This cluster of strains contained the LEE inserted into the 
chromosome at minute 82 . Subsequent branches of this cluster acquired the EAF plasmid and became EPEC strains while another branch acquired the bacteriophage encoding Stx as well as the pO157 plasmid found in $E$. coli $0157: \mathrm{H} 7$ and other STEC isolated from human disease.

\section{REFERENCES}

1. Benkel, P., M. Hudel, and T. Chakraborty. (1997): The localization of the LEE-region in the O26:H-Shiga toxin-producing Escherichia coli strain 413/ 89-1. 49th Annual Meeting of the Deutsche Gesellschaft für Hygiene und Mikrobiologie, Jena, Germany. P.53, abst. P196.

2. Deibel, C., S. Krämer, T. Chakraborty, and F. Ebel. (1998): EspE, a novel secreted protein of attaching and effacing bacteria, is directly translocated into infected host cells where it appears as a tyrosine-phosphorylated $90 \mathrm{kDa}$ protein. Molec. Microbiol. 28 : 463-474.

3. Elliott, E., Z. Li, C. Bell, D. Stiel, A. Buret, J. Wallace, I. Brzuszczak, and E. O'Loughlin. (1994): Modulation of host response to Escherichia coli O157 :H7 infection by anti-CD18 antibody in rabbits. Gastroenterol. 106:15541561.

4. Elliott, S., L.A. Wainwright, T. McDaniel, B. MacNamara, M. Donnenberg, and J.B. Kaper. (1998): The complete sequence of the locus of enterocyte effacement (LEE) from enteropathogenic Escherichia coli E2348/69. Molec. Microbiol. 28 : $1-4$.

5. Jarvis, K.G., J.A. Girón, A.E. Jerse, T.K. McDaniel, M.S. Donnenberg, and J.B. Kaper. (1995): Enteropathogenic Escherichia coli contains a specialized secretion system necessary for the export of proteins involved in attaching and effacing lesion formation. Proc. Natl. Acad. Sci. USA $92:$ 7996-8000.

6. Jarvis, K.G. and J.B. Kaper. (1996): Secretion of extracellular proteins by enterohemorrhagic Escherichia coli via a putative type III secretion system. Infect. Immun. 64 : 4826-2829.

7. Johnson, R.P., R.C. Clarke, J.B. Wilson, S.C. Read, K. Rahn, S.A. Renwick, K.A. Sandhu, D. Alves, M.A. Karmali, H. Lior, S.A. McEwen, J.S. Spika, and C.L. Gyles. (1996): Growing concerns and recent outbreaks involving non-O157:H7 serotypes of verotoxigenic Escherichia coli. J. Food Protect. 59 $: 1112-1122$.

8. Kaper, J.B., S. Elliott, V. Sperandio, N.T. Perna, G.F. Mayhew, and F.R. Blattner. (1998): Attaching and effacing intestinal histopathology and the locus of enterocyte effacement, p.163-182. In J.B. Kaper and A.D. O'Brien (eds.), Escherichia coli $\mathrm{O} 157: \mathrm{H} 7$ and Other Shiga Toxin-Producing E. coli Strains. American Society for Microbiology Press, Washington, D.C.

9. Kaper, J.B., L.J. Gansheroff, W.R. Wachtel, and A.D. O'Brien. (1998): Initmin-mediated adherence of Shiga toxin-producing Escherichia coli and attaching-and-effacing pathogens, p.148-156. In J.B. Kaper and A.D. O'Brien (eds.), Escherichia coli $\mathrm{O} 157: \mathrm{H} 7$ and Other Shiga Toxin-Producing E. coli Strains. American Society for Microbiology Press, Washington, D.C.

10. Kenny, B., R. DeVinney, M. Stein, D.J. Reinscheid, E.A. Frey, and B.B. 
Finlay. (1997): Enteropathogenic E. coli (EPEC) transfers its receptor for intimate adherence into mammalian cells. Cell $91: 511-520$.

11. Knutton, S., I. Rosenshine, M.J. Pallen, L. Nisan, B.C. Neves, C. Bain, C. Wolff, G. Dougan, and G. Frankel. (1998): A novel EspA-associated surface organelle of enteropathogenic Escherichia coli involved in protein translocation into epithelial cells. EMBO J. $17: 2166-2176$.

12. McDaniel, T.K., K.G. Jarvis, M.S. Donnenberg, and J.B. Kaper. (1995): A genetic locus of enterocyte effacement conserved among diverse enterobacterial pathogens. Proc. Natl. Acad. Sci. USA $92: 1664-1668$.

13. McDaniel, T.K. and J.B. Kaper. (1997): A cloned pathogenicity island from enteropathogenic Escherichia coli confers the attaching and effacing phenotype on E. coli K-12. Mol. Microbiol. 23 : 399-407.

14. Nataro, J.P. and J.B. Kaper. (1998): Diarrheagenic Escherichia coli. Clin. Microbiol. Rev. 11 : 142-201.

15. Perna, N.T., G.F. Mayhew, G. Pósfal, S. Elliott, M.S. Donnenberg, J.B. Kaper, and F.R. Blattner. (1998): Molecular evolution of a pathogenicity island from enterohemorrhagic Escherichia coli O157:H7. Infect. Immun. $66: 3810-3817$.

16. Philpott, D.J., D.M. McKay, W. Mak, M.H. Perdue, and P.M. Sherman. (1998): Signal transduction pathways involved in enterohemorrhagic Escherichia coli-induced alterations in T84 epithelial permeability. Infect. Immun. $66: 1680-1687$.

17. Rosenshine, I., M.S. Donnenberg, J.B. Kaper, and B.B. Finlay. (1992): Signal transduction between enteropathogenic Escherichia coli (EPEC) and epithelial cells: EPEC induces tyrosine phosphorylation of host cell proteins to initiate cytoskeletal rearrangement and bacterial uptake. EMBO J. $11: 3551-3560$.

18. Savkovic, S.D., A. Koutsouris, and G. Hecht. (1996): Attachment of a noninvasive enteric pathogen, enteropathogenic Escherichia coli, to cultured human intestinal epithelial monolayers induces transmigration of neutrophils. Infect. Immun. 64 : 4480-4487.

19. Savkovic, S.D., A. Koutsouris, and G. Hecht. (1997): Activation of NFkappaB in intestinal epithelial cells by enteropathogenic Escherichia coli. Am. J. Physiol. 273 : C1160-C1167.

20. Tzipori, S., I.K. Wachsmuth, C. Chapman, R. Birner, J. Brittingham, C. Jackson, and J. Hogg. (1986): The pathogenesis of hemorrhagic colitis caused by Escherichia coli $\mathrm{O} 157: \mathrm{H7}$ in gnotobiotic piglets. J. Infect. Dis. 154:712716.

21. Whittam, T.S. (1998): The evolution of Escherichia coli O157:H7 and other Shiga toxin-producing E. coli strains, J.B. Kaper and A.D. O'Brien (eds.), Escherichia coli O157:H7 and Other Shiga Toxin-Producing E. coli Strains. American Society for Microbiology Press, Washington, D.C.

22. Whittam, T.S., M.L. Wolfe, I.K. Wachsmuth, F. Orskov, I. Orskov, and R.A. Wilson. (1993): Clonal relationships among Escherichia coli strains that cause hemorrhagic colitis and infantile diarrhea. Infect. Immun. 61 : 1619-1629.

23. Wieler, L.H., T.K. McDaniel, T.S. Whittam, and J.B. Kaper. (1997): Insertion site of the locus of enterocyte effacement in enteropathogenic and 
enterohemorrhagic Escherichia coli differs in relation to the clonal phylogeny of the strains. FEMS Microbiol. Lett. 156:49-53.

24. Wolff, C., I. Nisan, E. Hanski, G. Frankel, and I. Rosenshine. (1998): Protein translocation into host epithelial cells by infecting enteropathogenic Escherichia coli. Molec. Microbiol. 28 : 143-155.

25. Yuhan, R., A. Koutsouris, S.D. Savkovic, and G. Hecht. (1997): Enteropathogenic Escherichia coli-induced myosin light chain phosphorylation alters intestinal epithelial permeability. Gastroenterol. 113 : 1873-1882. 